\title{
Correction to: Protective Effects of 28-O-Cafeoyl Betulin (B-CA) on the Cerebral Cortex of Ischemic Rats Revealed by a NMR-Based Metabolomics Analysis
}

\author{
Xia Liu' ${ }^{1}$ Zhi Ruan ${ }^{2} \cdot$ Xing-cheng Shao ${ }^{3,4} \cdot$ Hong-xuan Feng ${ }^{2} \cdot$ Lei Wu $^{2} \cdot$ Wei Wang $^{2} \cdot$ Hong-min Wang ${ }^{3}$. \\ Hong-yan $\mathrm{Mu}^{3,4} \cdot$ Ru-jun Zhang ${ }^{3} \cdot$ Wei-min Zhao ${ }^{3,4} \cdot$ Hai-yan Zhang ${ }^{2} \cdot$ Nai-xia Zhang $^{1}$ (1)
}

Published online: 16 January 2021

(c) Springer Science+Business Media, LLC, part of Springer Nature 2021

\section{Correction to: Neurochemical Research https://doi.org/10.1007/s11064-020-03202-z}

In the original version of this article, unfortunately the Fig. $3 \mathrm{c}$ and $\mathrm{f}$ were published with incorrect version. The correct version of the Fig. $3 \mathrm{c}$ and $\mathrm{f}$ are given below. This has been corrected by publishing this correction article. The original article has been corrected.

Publisher's Note Springer Nature remains neutral with regard to jurisdictional claims in published maps and institutional affiliations.

Xia Liu, Zhi Ruan, and Xing-cheng Shao contribute equally to this work.

The original article can be found online at https://doi.org/10.1007/ s11064-020-03202-z.

Wei-min Zhao

wmzhao@simm.ac.cn

$\triangle$ Hai-yan Zhang

hzhang@simm.ac.cn

$\triangle$ Nai-xia Zhang

nxzhang@simm.ac.cn

1 Department of Analytical Chemistry, Shanghai Institute of Materia Medica, Chinese Academy of Sciences,

Shanghai, China

2 CAS Key Laboratory of Receptor Research, Department of Pharmacology, Shanghai Institute of Materia Medica, Chinese Academy of Sciences, Shanghai, China

3 Department of Natural Product Chemistry, Shanghai Institute of Materia Medica, Chinese Academy of Sciences, Shanghai, China

4 University of Chinese Academy of Sciences, Beijing 100049, China 

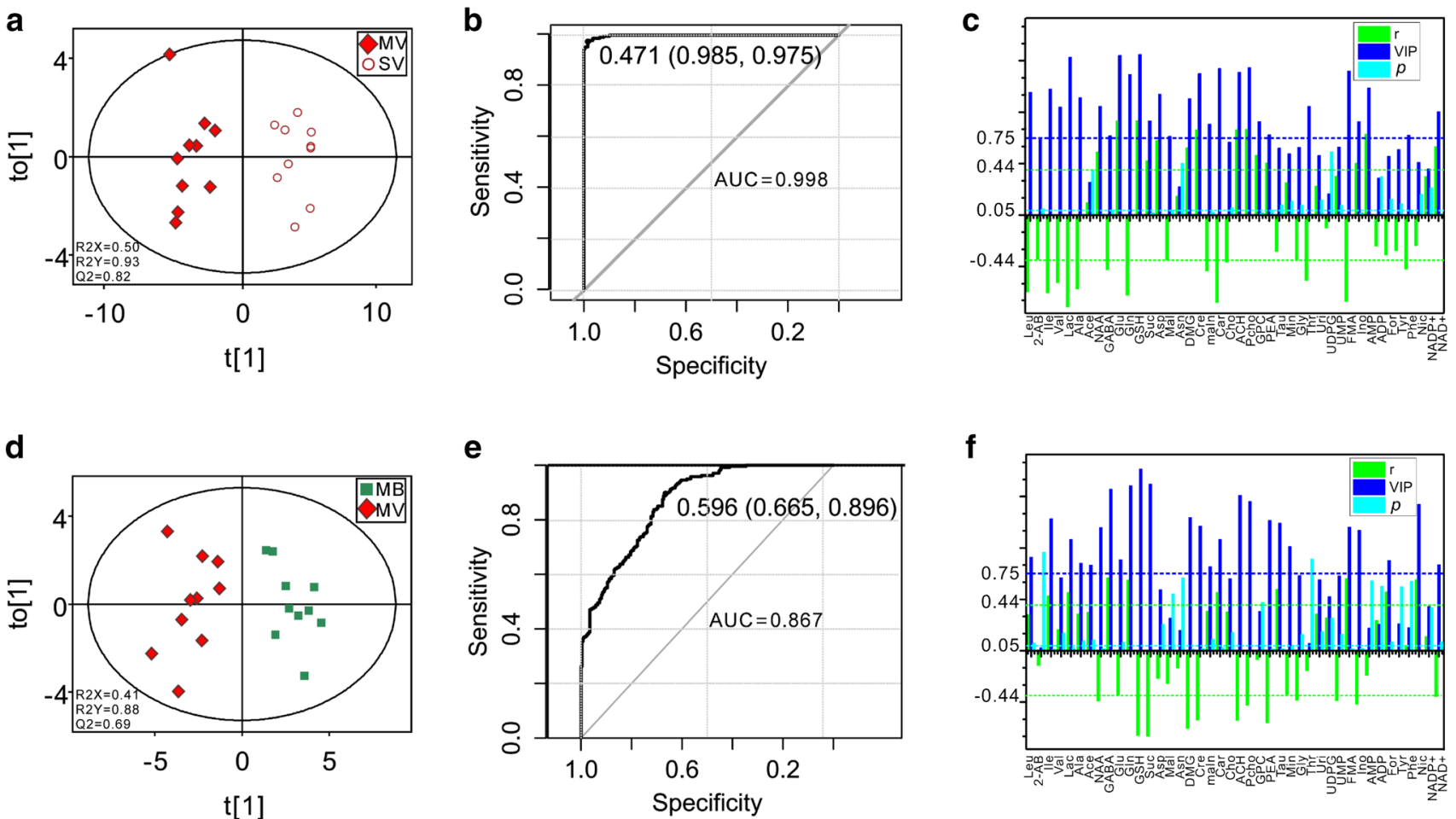

Fig. 3 Scores plots, ROC curves, and correlation coefficient and VIP plots derived from OPLS-DA models of the vehicle treated MCAO group ( $\mathrm{MV}, \mathrm{n}=10)$ vs the sham-vehicle group $(\mathrm{SV}, \mathrm{n}=10)$ samples $(\mathbf{a}-\mathbf{c})$, the B-CA-treated MCAO group $(\mathrm{MB}, \mathrm{n}=10)$ vs the vehicle-

treated MCAO group samples $(\mathrm{MV}, \mathrm{n}=10)(\mathbf{d}-\mathbf{f})$. The values of $\mathrm{Q} 2$ parameter in OPLS-DA scores plots, which were greater than 0.40 , coupling with the values of AUC parameter, which were greater than 0.500 , indicated that the established OPLS-DA models were valid 